\title{
Percutaneous Sacroplasty : Effectiveness and Long-Term Outcome Predictors
}

\author{
Jaehyung Lee, Eugene Lee, Joon Woo Lee, Yusuhn Kang, Joong Mo Ahn, Heung Sik Kang \\ Department of Radiology, Seoul National University Bundang Hospital, Seongnam, Korea
}

Objective : To evaluate the effectiveness and long-term outcome predictors of percutaneous sacroplasty (PSP).

Methods : This single-center study assessed 40 patients with sacral insufficiency fractures using the short-axis technique under C-arm flat-panel detector computed tomography (CT). Two radiologists reviewed the patients' magnetic resonance and CT images to obtain imaging findings before PSP and determine technical success, respectively. The short-term outcomes were visual analog scale score changes and opioid usage reductions. Long-term outcomes were determined using telephone interviews and the North American Spine Society (NASS) patient-satisfaction index at least one year after PSP.

Results : Technical success was achieved without any significant complications in 39 patients (97.5\%). Telephone interviews were possible with 12 patients and failed in 10 patients; death was confirmed in 18 patients. Fifteen patients (50\%) re-visited the hospital and received conservative treatment, including spinal injections. Nine patients reported positive satisfaction (NASS patientsatisfaction index 1 or 2), while the negative satisfaction group (NASS patient-satisfaction index 3 or $4, n=3$ ) showed a higher incidence of compression fractures at the thoracolumbar spine level (66.7\% vs. $22.2 \%)$ and previous spinal injection history $(66.7 \%$ vs. $33.3 \%)$. The poor response group also showed higher incidences of facet joint arthrosis (100\% vs. $55.6 \%)$, central canal stenosis (100\% vs. $22.2 \%$ ), neural foraminal stenosis (33.3\% vs. $22.2 \%$ ), scoliosis (100\% vs. $33.3 \%$ ), and sagittal malalignment (100\% vs. $44.4 \%)$.

Conclusion : PSP was effective for sacral insufficiency fractures and showed good long-term outcomes. Combined compression fractures in the thoracolumbar spine and degenerative lumbar pathologies could be possible poor outcome predictors.

Key Words : Sacrum · Fractures · Fluoroscopy $\cdot$ Vertebroplasty $\cdot$ Spinal stenosis.

\section{INTRODUCTION}

Sacral insufficiency fractures (SIFs) resulting from osteopenia, osteolysis from cancer metastasis, or minor trauma can be severely debilitating for elderly patients and may be exacerbated by delays in diagnosis and limited treatment options ${ }^{10,14,19)}$. Conservative treatments, including pain medica- tion, bed rest, and physical therapy can improve pain and functional disability. However, these treatments may necessitate prolonged hospitalization and the extended use of care and hospice care services. Surgical treatment also has limited applicability in this largely elderly population, since reliable hardware implantation may be impossible within the bone that has been significantly weakened by osteoporosis or infil-

\footnotetext{
- Received : January 17, 2020 •Revised : February 24, 2020 •Accepted : March 3, 2020

- Address for reprints : Eugene Lee

Department of Radiology, Seoul National University Bundang Hospital, 82 Gumi-ro, 173beon-gil, Bundang-gu, Seongnam 13620, Korea Tel : +82-31-787-7627, Fax : +82-31-787-4011, E-mail : eugene801027@gmail.com, ORCID : https://orcid.org/0000-0003-4205-2362
}

This is an Open Access article distributed under the terms of the Creative Commons Attribution Non-Commercial License (http://creativecommons.org/licenses/by-nc/4.0) which permits unrestricted non-commercial use, distribution, and reproduction in any medium, provided the original work is properly cited. 
trative osteolytic lesions ${ }^{10)}$.

Percutaneous sacroplasty (PSP) is a minimally invasive procedure in which bone cement (polymethyl methacrylate, PMMA) is injected into the fractured sacrum; PSP is indicated for patients who do not respond to conservative therapy or are not surgical candidates. Recent studies have suggested that PSP is an effective treatment approach for both osteoporotic and metastatic SIFs, and patients experience nearly full pain relief and mobility improvement with good short-term outcomes $^{10,14,19)}$.

Most of the articles published to date have discussed the technical feasibility or short-term clinical outcomes of PSP $^{4,10,11,14)}$. Only one recent report by Frey et al. ${ }^{6}$ focused on the long-term outcome of this procedure in a 10 -year prospective, observational cohort study. This study mainly assessed the long-term efficacy of sacroplasty versus non-surgical management in treating SIFs, including the effect on pain relief, usage of opioids and other analgesics, patient satisfaction, and complication rates.

Therefore, the current study aimed to investigate the natural long-term clinical outcomes of PSP. Furthermore, this study aimed not only to evaluate the technical success or satisfaction of patients after the procedure but also to investigate the factors that may affect the differences in satisfaction levels.

\section{MATERIALS AND METHODS}

\section{Study group}

This study was approved by the Institutional Review Board of Seoul National University Bundang Hospital (IRB No. B-1902-522-103). Considering the use of anonymized patient data, the requirement for informed consent was waived. From April 2006 to March 2018, we enrolled 43 patients who were referred for PSP to our spinal intervention center at a single tertiary hospital. All patients complained of very severe low back or buttock pain without a definite neurologic deficit. Since the aim of our study was to investigate the long-term outcomes of PSP, three patients were excluded because they had metastatic sacral fractures related to an underlying malignancy (lung cancer, cervical cancer, and renal cell carcinoma). Finally, a total of 40 patients (male : female, $4: 36$; mean age, $75.6 \pm 7.9$ years; range, 59-89 years) with benign SIFs were enrolled (Fig. 1). Our institution basically followed the procedure guidelines of percutaneous vertebroplasty, which applied to the patients with acute sacral fracture (evident on magnetic resonance imaging [MRI]) with severe pain, despite enough conservative treatment for more than 2 weeks. However, the procedure was performed quickly in three cases, when the pain was too severe despite the conservative management in short-term. For pain relief, our institution used drugs or steroid injection treatment before the procedure. Most patients tried pain control with opioid drugs $(n=24)$ or non-opioid drugs $(n=10)$, but five patients received steroid injection therapy (transforaminal epidural steroid injection $[n=2]$ and caudal injection $[n=3])$.

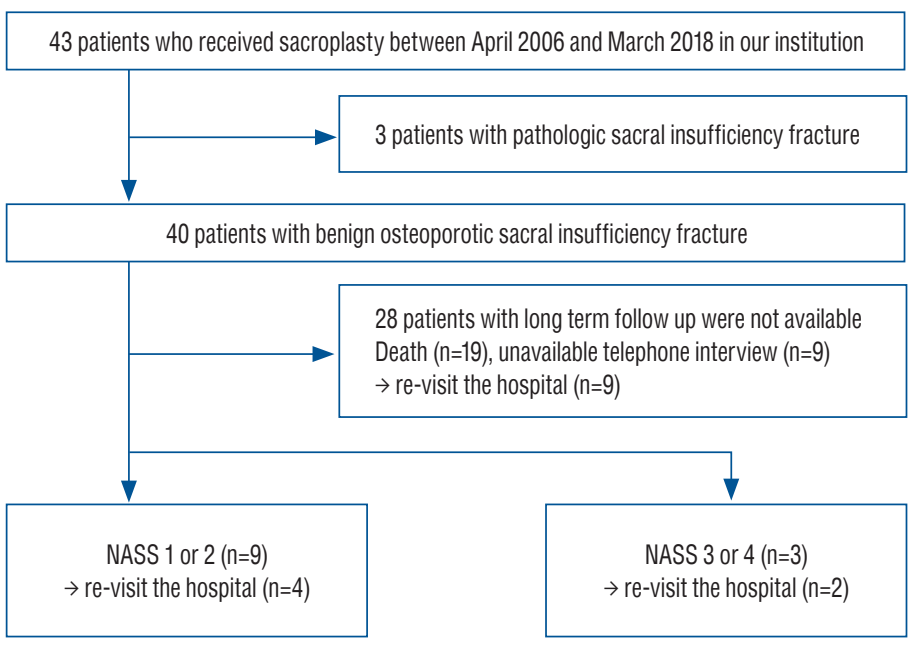

Fig. 1. Flow chart depicting the process of patient selection. NASS : North American Spine Society. 


\section{Clinical assessment}

The review of the patients' medical records was performed by one of the authors who was not involved in the patients treatment. Information regarding each patient's age, sex, dualenergy X-ray absorptiometry (DEXA) score, cause of sacral fracture, hospital stay, short-term follow-up with overall pain relief, and any comorbidities that could be related to the patient's prognosis was collected.

Short-term outcomes were assessed either by the orthopedic surgeon who initially interviewed the enrolled patients or by the radiologist who performed the PSP. Follow-up assessments were performed at least within 1 month after the procedure. The grade of satisfaction was categorized considering the patients' subjective responses or the visual analog scale (VAS) score. A reduction of more than 3 points or 30\% symptom relief was categorized as much improved, reductions of less than 3 points or $30 \%$ symptom relief were categorized as slightly improved, and others were categorized under the no-change or aggravated groups. Analgesic use was described as opioid use. If opioids were used before the procedure, we checked whether the opioid was continued or not at the time of discharge.

Interviews to assess long-term outcomes were conducted with all patients approximately at 1 year to 13 years (mean, 6.7 \pm 3.4 years; range, 1.1-13.2 years) after the end of the procedure; in these interviews, the degree of pain relief and response associated with the procedure were evaluated. On the basis of the 4-point North American Spine Society (NASS) patient satisfaction index ${ }^{1)}$, patients were asked to recall their overall level of pain improvement as follows : 1 , the sacroplasty met my expectations; 2, I experienced less improvement than I had hoped, but I would undergo the same procedure again for the same result; 3 , the sacroplasty helped, but I would not undergo the same procedure again for the same result; and 4, my condition is the same or worse than that before the sacroplasty. Then, we categorized the four possible responses as a "good response" (scored with 1 or 2 points) or "poor response" (scored with 3 or 4 points). We also investigated any re-visit history and other comorbidities during the long-term followup. If the patient's pain recurred, the number of visits to the hospital and the treatment (conservative, medical treatment, or steroid injection) they received were also checked. If the patient had died, the above-mentioned items were confirmed by the family members.

\section{PSP with C-arm flat-panel detector computed tomography (CT)}

Each sacroplasty was performed by two radiologists with 7 and 16 years' experience in spine intervention. Before PSP, MRI images were carefully reviewed to decide the appropriate needle target site and needle tract. In the angiography room, all patients were positioned prone on the table and were conscious under local anesthesia with 2\% lidocaine (Huons, Hwaseong, Korea). Using fluoroscopic guidance with an oblique trajectory, real-time positioning of one trocar needle (Cook : osteo-site bone biopsy needle, 11-gauge) was performed at the fracture site at one side of the sacral ala along the short axis of the sacrum parallel to the sacroiliac joint (Fig. 2).

C-arm CT was then performed by rotating the angiographic (fluoroscopic) tube $240^{\circ}$ to check the needle tip position. If the needle was placed at the fracture site and did not penetrate the anterior cortex, liquid and powder PMMA (DePuy International, Leeds, UK) mixed with $500 \mathrm{mg}$ of cefazolin were used as bone cement. The cement was then injected under realtime fluoroscopic guidance, as in conventional vertebroplasty. The injection was stopped if the cement spread near the sacral foramen or outside the anterior cortex. C-arm CT was then performed to check the cement distribution. If cement distribution was appropriate, the same method was also performed for the contralateral sacral ala. After cement injection, C-arm CT was performed again to check the cement distribution. The amounts of cement injected were recorded ${ }^{12)}$.

\section{Image analysis}

Two radiologists (one with a 1-year fellowship in musculoskeletal imaging and an attending radiologist with 7 years' experience in musculoskeletal imaging) who were blinded to the clinical outcome reviewed the pre-procedural patients' MR images taken within one month of the occurrence of symptoms. We reached a consensus regarding the types and locations of sacral fractures, whether there was an anterior cortex breakage, neural foraminal extension, sagittal malalignment, visible fracture gap, and other compression fractures at the TL spine levels. The types and locations of sacral fractures were categorized according to the AOSpine Sacral Fracture Classification (Table 1$)^{3,20)}$.

For evaluation of factors that can affect the patient's outcome, we also evaluated the degree of degenerative changes in the lower lumbar spine. Based on the recent radiographic 

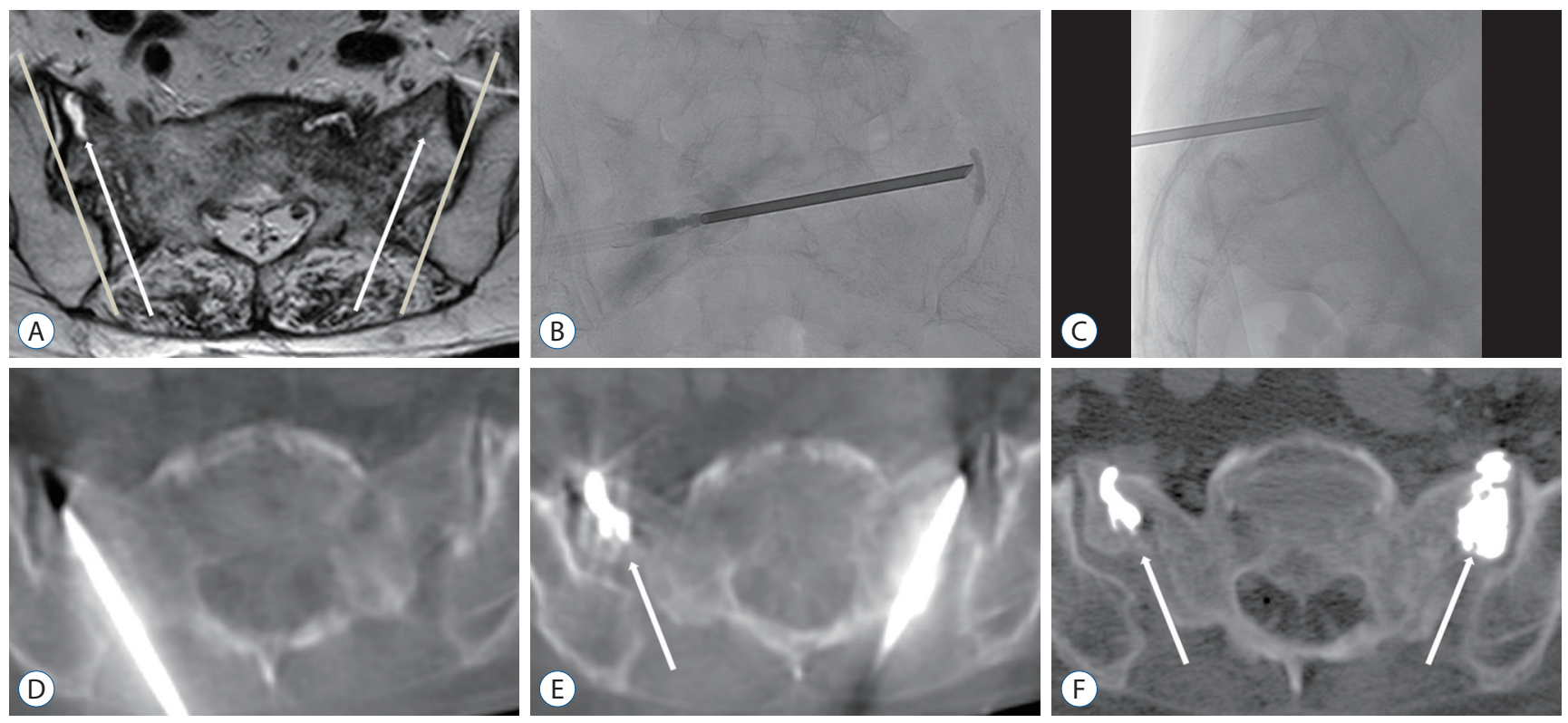

Fig. 2. A 72-year-old woman with sacral insufficiency fracture extension to both sacral ala, treated with the short-axis approach. A : Axial T2-weighted image shows fracture gaps with perilesional edema of the bilateral alae. We planned to place a needle at the fracture gap along the short axis of the sacrum parallel to the sacroiliac joint (arrows : the direction of the trocar needle, line : sacroiliac joint). B and C: A needle was inserted into the target site under fluoroscopy. D : C-arm flat-panel detector CT (C-arm CT) can check the precise needle position at a fracture site. The needle tip is shown within the sacrum with no violation of the anterior cortex of the sacrum. E and F: Each CT image demonstrates cement distribution. It shows near total cement filling at both SIF sites (arrows), without any cement leakage. CT: computed tomography.

Table 1. AO sacral fracture classification system

\begin{tabular}{|c|c|c|}
\hline Type A : lower sacrococcygeal injuries & Type B : posterior pelvic injuries & Type C : spinopelvic injuries \\
\hline $\begin{array}{l}\text { A1: Coccygeal or compression injuries as well as } \\
\text { ligamentous avulsion fractures } \\
\text { A2: Non-displaced transverse fractures below } \\
\text { the SI joint } \\
\text { A3 : Displaced transverse fractures below the SI } \\
\text { joint }\end{array}$ & $\begin{array}{l}\text { B1 : Longitudinal fracture medial to the foramen } \\
\text { B2 : Longitudinal fracture lateral to the foramen } \\
\text { B3 : Longitudinal injury thought the foramen }\end{array}$ & $\begin{array}{l}\text { C0 : Non displaced sacral U fracture (commonly } \\
\text { seen in low energy insufficiency fractures) } \\
\text { C1: Any unilateral B-subtype where the ipsilateral } \\
\text { superior } \$ 1 \text { facet is discontinuous with the } \\
\text { medial portion of the sacrum } \\
\text { C2 : Bilateral complete B type fracture without a } \\
\text { transverse component } \\
\text { C3: Displaced sacral U type fracture }\end{array}$ \\
\hline
\end{tabular}

SI : sacroiliac

grading systems for the lumbar spine ${ }^{16,17)}$, we analyzed whether the patients showed moderate to severe central or neural foraminal stenosis at the L4-5 or L5-S1 levels. Evaluation of facet joint arthrosis was based on the radiographic methods reported by Pathria et al. ${ }^{18)}$, Grogan et al. ${ }^{9}$, and Weishaupt et al. ${ }^{21)}$. We reviewed whether the patient had lumbar scoliosis and sagittal malalignments such as spondylolisthesis or retrolisthesis.

Assessment of technical success with CT was performed immediately after PSP and was defined by the following criteria: good cement filling (more than 75\%) at the fracture site and no evidence of major symptomatic cement leakage ${ }^{12)}$. After the procedure, we confirmed that there was no complication, including cement extravasation (anteriorly through the nutritional vessels, posteriorly into the spinal canal, medially into the neuroforamina, laterally into the sacroiliac joint, cephalad into the intervertebral space or at least into the fracture), infections, hemorrhages, pulmonary emboli, or procedure-related deaths during any period of follow-up ${ }^{2,10)}$.

\section{Statistical analysis}

No formal statistical analysis was performed to analyze the 
Table 2. Baseline characteristics and short-term clinical outcomes in patients who received sacroplasty for sacral insufficiency fractures $(n=40)$

\begin{tabular}{lcc}
\hline & Value & p-value* \\
\hline Age (years) & $75.6 \pm 0.9$ \\
Sex & $4(10.0)$ \\
Male & $36(90.0)$ \\
Female & $-3.88 \pm 0.92$ \\
DEXA score (T-score) & \\
Cause of sacral fractures & $17(42.5)$ \\
None & $22(55.0)$ \\
Slip down & $1(2.5)$ \\
Trauma & $1.66 \pm 1.27$ \\
Hospital stay (days) & \\
VAS score & $9.11 \pm 1.23$ \\
Pre-treatment & $2.53 \pm 1.91$ \\
Post treatment & \\
Opioid usage & $24(64.8)$ \\
Pre-treatment & $3(8.1)$ \\
Post treatment & 28 \\
Overall pain relief & 7 \\
Much improved & 2 \\
Slightly improved & \\
No change & $<0.001$ \\
\hline
\end{tabular}

Values are presented as mean \pm standard deviation or number (\%). *Analyzed using the Wilcoxon Rank Sum Test. DEXA : dual-energy X-ray absorptiometry, VAS : visual analog scale

prognostic value of the clinical or imaging characteristics. Improvements in the VAS scores and reductions in opioid use on short-term follow-up were analyzed using the Wilcoxon Rank Sum Test. The percentage of the effect of the predicted factors in the good and bad response groups was presented. All statistical analyses were performed using SPSS version 21.0. (SPSS Inc., IBM Company, Chicago, IL, USA). Two-sided $p$-values $<0.05$ were considered statistically significant.

\section{RESULTS}

\section{Clinical and imaging characteristics}

Clinical characteristics of the included 40 patients (mean age \pm standard deviation [SD], $75.6 \pm 7.9$ years; female : male, $36: 4$ )
Table 3. Imaging findings in 40 patients who received sacroplasty by using the short-axis technique

\begin{tabular}{lc}
\hline & Value \\
\hline Imaging findings related to sacral fracture & \\
Types of SIF (AO classification) & $3(7.5)$ \\
B2 & $33(82.5)$ \\
C0 & $4(10.0)$ \\
C2 & $26(65.0)$ \\
Anterior cortex breakage & $6(15.0)$ \\
Neural foraminal extension & $8(20.0)$ \\
Sagittal malalignment of fracture segment & $37(92.5)$ \\
Fracture gap & \\
Imaging findings related to combined fractures or & \\
degenerative changes & $14(35.0)$ \\
Compression fractures of T-L spine level & $22(55.0)$ \\
Lumbar facet joint arthrosis (L4-5 or L5-S1 level) \\
Central canal stenosis (L4-5 or L5-S1 level) & $10(25.0)$ \\
$\quad$ Neural foraminal stenosis (L4-5 or L5-S1 level) & $10(25.0)$ \\
Lumbar scoliosis & $15(37.5)$ \\
Sagittal malalignment of lumbar spine & $18(45.0)$ \\
(spondylolisthesis or retrolisthesis) & \\
\hline
\end{tabular}

Values are presented as number (\%). SIF : sacral insufficiency fracture

and the number of cases that could be observed for improvement of pain are summarized in Table 2. Most patients had a fracture without any specific cause $(n=17,42.5 \%)$, but some patients had minor trauma such as slips $(n=22,55 \%)$ or a blunt trauma from the traffic accident $(\mathrm{n}=1,2.5 \%)$. The mean $\pm \mathrm{SD}$ of the duration of hospitalization after the procedure was $1.66 \pm 1.27$ days. Only 29 of the 40 patients had result of DEXA exam according to our institutional chart. T-scores of each 29 patients were all below -2.5 (mean \pm SD, $-3.88 \pm 0.92)$ and met the diagnosis for osteoporosis.

Imaging findings of the patients who received sacroplasty are summarized in Table 3. According to the AOSpine Sacral Fracture Classification ${ }^{3,20)}$, type C0 was seen most frequently (33 of 40 patients, $82.5 \%$ ), followed by type C2 (four of 40 patients, 10\%), and type B2 (three of 40 patients, 7.5\%). Anterior cortex breakage was noted in 26 of 40 patients (65\%). Neural foraminal extension was seen in six of 40 patients (15\%). Sagittal malalignment was visible in eight of 40 patients (20\%). Fracture gap was seen in 37 of 40 patients (92.5\%). Combined compression fractures of the T-L spine level were seen in 14 of 40 patients (35\%). 


\section{Technical success}

Sacroplasties were performed on unilateral $(n=3)$ or bilateral sacral alae $(n=37)$ in all patients, and an average of $2.6 \mathrm{~mL}$ (range, 1-5) of cement was injected at each sacral fracture site. Technical success was achieved in almost all patients $(n=39$, $97.5 \%$ ) without any significant complication. Only one patient reported left radicular pain after the procedure. Mild cement leakage over the fractured anterior cortex led to irritation of the left L5 nerve root. The patient received epidural steroid injection through an extraforaminal targeting approach at the L5/S1 level (Fig. 3). The patient's pain successfully improved after injection without symptom recurrence on final follow-up.

\section{Short-term treatment outcomes}

Thirty-seven patients (92.5\%) were available for short-term follow-up assessments. Among these patients, 28 (75.7\%) reported a much improved and seven patients (18.9\%) reported a slightly improved status after sacroplasty. Only two patients (5.4\%) had persistent symptoms. The mean VAS score was $9.11 \pm 1.23$ pre-treatment, which decreased to $2.53 \pm 1.91$ posttreatment $(p<0.001)$. The number of patients requiring opioid treatment decreased from $37(64.8 \%)$ to three (8.1\%) postoperatively $(p<0.001)$.

\section{Long-term clinical outcomes}

The long-term outcomes are summarized in Table 4. Of the 40 patients, telephone interviews were possible with 12 patients. Among the remaining 28 patients, death was confirmed in 18 patients and follow-up loss in 10 patients.

Of the 12 patients for whom long-term clinical outcome data were obtained, nine (75\%) replied that they had experi- enced positive satisfaction (NASS patient-satisfaction index 1 or 2). The negative satisfaction group (NASS patient-satisfaction index 3 or 4 ) showed a higher incidence of compression fractures at the T-L-spine level on initial MR imaging and a greater re-visit rate than the positive satisfaction group : $66.7 \%$ vs. $22.2 \%$ for compression fracture incidence and $66.7 \%$ vs. $33.3 \%$ for the re-visit rate.

The death group showed a higher incidence of comorbidity than the survival group : $83.3 \%$ vs. $25.0 \%$. Chronic medical diseases such as hypertension, chronic kidney disease, dyslipidemia, myocardial infarction, stroke, liver cirrhosis, rheumatic disease, and chronic obstructive pulmonary disease were the most frequent comorbidities $(n=14)$, followed by malignancy $(n=8)$ and infection $(n=3)$.

Table 4. Long-term clinical outcomes in patients who received sacroplasty for sacral insufficiency fractures $(n=40)$

\begin{tabular}{lc}
\hline & Value \\
\hline $\begin{array}{l}\text { Long term follow up* with NASS patient-satisfaction } \\
\text { index }\end{array}$ & 9 \\
Good response (NASS 1 or 2) & 3 \\
Poor response (NASS 3 or 4) & 18 \\
Death & 10 \\
Follow up loss & \\
Additional items in NASS patient-satisfaction & $9(75)$ \\
questionnaire & \\
Wanted to repeat the sacroplasty in a similar attack \\
$\quad$ of LBP \\
Recommend the same procedure to others
\end{tabular}

Values are presented as number (\%). *At least 1 year after the procedure date by using formatted questions including the NASS patientsatisfaction index. ${ }^{\dagger}$ Measurable in 12 patients. NASS : North American Spine Society, LBP : low back pain
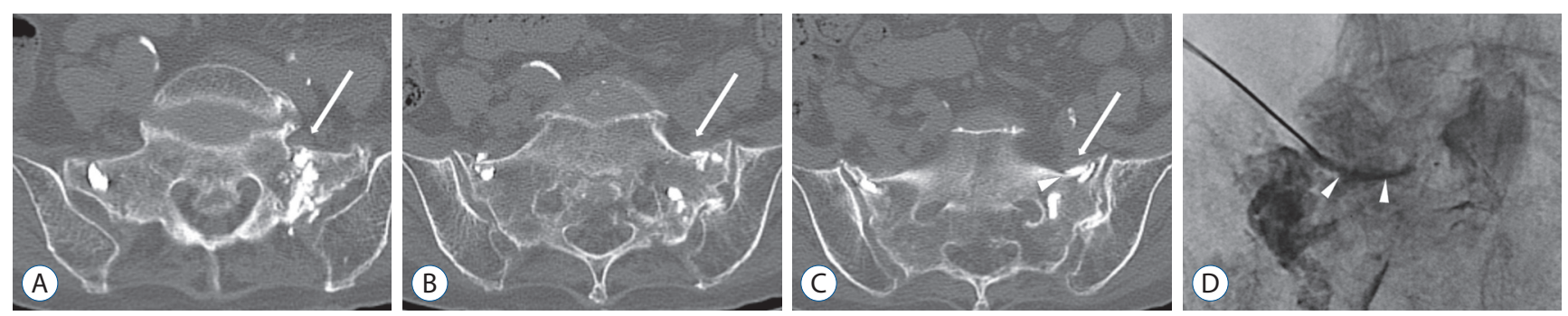

Fig. 3. An 81-year-old woman complaining of left side radicular pain after the procedure. A-C: Axial image of pelvis computed tomography shows mild cement leakage (arrowhead) over the fractured anterior cortex led to irritation of the left L5 nerve root (arrows). D: The patient received transforaminal epidural steroid injection through the extraforaminal targeting approach at the L5/S1 level. Anteroposterior fluoroscopic image shows that injected contrast (arrowheads) flows along the medial margin of the pedicle. 
For the additional items in the questionnaire, nine patients (75\%) replied that they would undergo a repeat PSP if they experienced a similar attack of low back pain (LBP) and all 12 patients replied that they would recommend the same procedure to others.

Among the 30 patients with available long-term follow-up medical data (NASS 1 or 2, NASS 3 or 4, or death), $15(50 \%)$ re-visited the hospital after the procedure. The frequency of re-visits to the hospital was higher in the poor response group than in the good response group : $67 \%$ vs $44 \%$. The causes of re-visits were not related to the previous sacral fracture or PSP, but were mostly new-onset LBP due to degeneration ( $\mathrm{n}=12$, $80 \%$ ) and new compression fractures in another vertebral segment $(n=3,20 \%)$. The patients were treated conservatively with modalities including spinal injections $(\mathrm{n}=8,60 \%)$, medi-

Table 5. Comorbidity, cause, and treatment during re-visits in the good response group (NASS 1 or 2), poor response group (NASS 3 or 4), and death group in the long-term follow up $(n=30)$

\begin{tabular}{|c|c|c|c|}
\hline & $\begin{array}{c}\text { Good } \\
\text { response } \\
\text { (NASS } 1 \text { or } 2) \\
(n=9)\end{array}$ & $\begin{array}{c}\text { Poor } \\
\text { response } \\
\text { (NASS } 3 \text { or } 4) \\
(n=3)\end{array}$ & $\begin{array}{l}\text { Death } \\
(n=18)\end{array}$ \\
\hline Age (years) & $75.6 \pm 8.4$ & $76.6 \pm 9.2$ & $75.5 \pm 8.2$ \\
\hline \multicolumn{4}{|l|}{ Sex } \\
\hline Male & 0 & 0 & 3 \\
\hline Female & 9 & 3 & 15 \\
\hline Revisit & $4(44)$ & $2(67)$ & $9(50)$ \\
\hline \multicolumn{4}{|l|}{ Cause of revisit } \\
\hline Degeneration & 3 & 2 & 7 \\
\hline New compression fracture & 1 & 0 & 2 \\
\hline \multicolumn{4}{|l|}{ Treatment on the revisit } \\
\hline Observation & 1 & 0 & 4 \\
\hline Medication & 0 & 0 & 2 \\
\hline Spinal injection & 3 & 2 & 3 \\
\hline \multicolumn{4}{|l|}{ Comorbidity } \\
\hline Malignancy & 0 & 1 & 7 \\
\hline Chronic medical disease ${ }^{*}$ & 1 & 1 & 12 \\
\hline Infection & 0 & 0 & 3 \\
\hline None & 8 & 1 & 3 \\
\hline
\end{tabular}

Values are presented as mean \pm standard deviation or number (\%). *HTN, CKD, dyslipidemia, myocardial infarction, stroke, liver cirrhosis, rheumatic disease, COPD. NASS : North American Spine Society, HTN : hypertension, CKD : chronic kidney disease, COPD : chronic obstructive pulmonary disease cation $(\mathrm{n}=2,13 \%)$, and observation $(\mathrm{n}=5,33 \%)$ (Table 5).

In terms of degenerative changes in pre-procedural MRI, the poor response group showed a higher incidence of facet joint arthrosis (100\% vs. $55.6 \%)$, central canal stenosis (100\% vs. $22.2 \%$ ), neural foraminal stenosis (33.3\% vs. $22.2 \%$ ), scoliosis (100\% vs. $33.3 \%)$, and sagittal malalignment (100\% vs. $44.4 \%$ ) than the good response group (Table 6).

\section{DISCUSSION}

The incidence of SIFs is increasing within the rapidly growing population of elderly people. According to the $2017 \mathrm{Popu}-$ lation dynamics and dynamics trend in the Korean Statistical Information Service, the average life expectancy of Koreans is 82.7 years (79.7 years in males and 85.7 years in females) ${ }^{13}$. In our study, the mean age of patients receiving sacroplasty was $75.6 \pm 7.9$ years. Thus, the results indicate that the remaining life expectancy of the patients after the procedure is not high. Indeed, 18 of the 40 patients (45\%) died during long-term follow-up. Therefore, short-term satisfaction after PSP for the rest of the patient's life is an important aspect. However, an even more important but frequently overlooked aspect is the determination of factors that may affect the patient's satisfac-

Table 6. Imaging findings related to fractures or degeneration in the good response group (NASS 1 or 2) and poor response group (NASS 3 or 4) in long-term follow-up ( $n=12)$

\begin{tabular}{lcc}
\hline & $\begin{array}{c}\text { Good response } \\
\text { (NASS 1 or 2) } \\
(\mathbf{n}=9)\end{array}$ & $\begin{array}{c}\text { Poor response } \\
\text { (NASS 3 or 4) } \\
(\mathbf{n}=3)\end{array}$ \\
\hline $\begin{array}{l}\text { Compression fractures of T-L spine } \\
\text { level }\end{array}$ & $2(22.2)$ & $2(66.7)$ \\
$\begin{array}{l}\text { Lumbar facet joint arthrosis (L4-5 } \\
\text { or L5-S1 level) }\end{array}$ & $5(55.6)$ & $3(100.0)$ \\
$\begin{array}{l}\text { Central canal stenosis (L4-5 or } \\
\text { L5-S1 level) }\end{array}$ & $2(22.2)$ & $3(100.0)$ \\
$\begin{array}{l}\text { Neural foraminal stenosis (L4-5 or } \\
\text { L5-S1 level) }\end{array}$ & $2(22.2)$ & $1(33.3)$ \\
$\begin{array}{l}\text { Lumbar scoliosis } \\
\begin{array}{l}\text { Sagittal malalignment of lumbar } \\
\text { spine (spondylolisthesis or } \\
\text { retrolisthesis) }\end{array}\end{array}$ & $3(33.3)$ & $3(100.0)$ \\
\hline
\end{tabular}

Values are presented as number (\%). NASS : North American Spine Society 
tion with the procedure during the remaining life expectancy over the course of long-term follow-up.

Most studies in the literature have already described good short-term outcomes with pain relief. They also provide clinical evidence of the safety and efficacy of sacroplasty for the treatment of SIFs. Patients with SIFs due to osteoporosis, cancer-related fractures, or minor trauma reported complete resolution or improvement in overall pain, and the benefits persisted following the sacroplasty procedures ${ }^{5,10,14)}$. According to recent studies, the predictive factors for the short-term clinical outcome of sacroplasty can be summarized into three categories; VAS score for representing pain, patient mobility, and analgesic usage.

Kortman et al. ${ }^{14)}$ included 243 patients with an average pretreatment VAS score of $9.2 \pm 1.1$ and found a significant improvement after sacroplasty to $1.9 \pm 1.7$ in patients with SIFs. In the study by Eichler et al. ${ }^{4}$, the mean VAS score decreased from $8.1 \pm 1.9$ to a mean value of $3.1 \pm 1.2$ in 12 patients. Gupta et al. ${ }^{10)}$ used the Functional Mobility Scale to represent the procedural effect on mobility and ambulation. The average preprocedure score of $3.0(2.0-3.0)$ decreased to $1.0(0.25-2.8)$. Kamel et al. ${ }^{11)}$ noted a significant reduction in the use of both narcotics and non-narcotics. Among these predictive factors, we measured changes in VAS score and opioid usage to indicate short-term outcomes. The results showed much improved or slightly improved pain relief in almost all patients, as well as decreased mean VAS scores and opioid usage for analgesia. Specifically, the mean VAS score was $9.11 \pm 1.23$ pre-treatment and it decreased to $2.53 \pm 1.91$ post-treatment. Opioid usage also decreased from $64.8 \%$ pre-operatively to $8.1 \%$ postoperatively. These results well correspond to those of previous studies.

With respect to long-term outcomes, we reviewed recent papers published from 2008 to 2018 by performing a literature search in Medline via PubMed. Only four studies had followup periods of up to 1 year, ${ }^{4,6,7,14)}$. The study by Gjertsen et al. ${ }^{7)}$ was the first to perform long-term follow-up between 4 to 12 months after sacroplasty. However, it was limited in that it presented only the subjective pain relief effect of the long-axis technique and did not use objective indicators. Recent studies by Kortman et al. ${ }^{14)}$ and Eichler et al. ${ }^{4)}$ showed a significant decrease in the VAS score during follow-up periods over 1 year after sacroplasty. Furthermore, Frey et al. .) $^{6}$ performed a 10year prospective study of 244 patients and showed a signifi- cant reduction in opioid usage as well as VAS scores in the sacroplasty group compared to the control group. These studies are similar in that they describe objective indicators of the long-term efficacy of sacroplasty. We attempted to numerically present the results of surveys by telephone interviews (NASS patient-satisfaction index) instead of the VAS score, as objective indicators. The good response group was three times larger than the group showing a poor response, which is consistent with the evidence of good long-term efficacy of sacroplasty in the previous studies. However, the patients may experience a recurrence in pain and may have to re-visit the hospital. Moreover, the patient's satisfaction with the procedure is not good in some cases. The studies mentioned above did not consider the prognostic factors that may affect the patient's satisfaction with the procedure over the short life expectancy of the patient after the procedure.

The radiologic features involving the intervertebral discs, end plates, bone marrow changes, facet joints, and the spinal canal in relation to the pathogenesis of degenerative changes in the spine are associated with LBP and reduced physical function. The pathogenesis of this degenerative process represents a biomechanically related continuum of alterations, which can be identified with different imaging modalities, ${ }^{8,15)}$. Therefore, we hypothesized that degenerative changes as well as fractures seen in the patient's image modalities at the time of the procedure may affect the long-term outcome by causing LBP or buttock pain in patients. Indeed, in our study, the poor response group showed a higher incidence of combined Tand L-spine fractures and degenerative changes, including central canal stenosis, facet joint arthrosis, scoliosis, and sagittal malalignment. The positive satisfaction group showed a lower incidence of hospital re-visits for spinal injection than the negative satisfaction group. In the latter group, more degenerative changes in MRI were likely to result in a re-visit to the hospital for pain control. Thus, patient groups showing degenerative changes that can be seen on pre-operative images are more likely to return to the hospital or experience less satisfaction after the procedure than those who do not. The difference in the incidence between the two groups is considered to affect the quality of the patient's remaining life.

In fact, the presentation of pain caused by degenerative changes is likely to overlap with the pain caused by sacral fracture. The results of our study support the fact that degenerative changes and sacral fractures affect the VAS score or satis- 
faction scores of patients simultaneously. For long-term results, since the healing of the fracture site after the PSP is almost complete, the actual long-term outcome may be dependent on degenerative lumbar pathologies, as shown in this result. Thus, the poor prognosis in the presence of combined compression fractures is thought to be due to the subsequent spine problem rather than the compression fracture itself.

There are several limitations to our research. First, we reviewed the previous electronic medical records and collected the available data. Therefore, VAS scores and opioid usage or disability index data for long-term follow-up were not available in most of the cases. To overcome this, we used the NASS satisfaction index scale through telephone interviews. Second, many patients died during the long-term follow-up and the number of patients decreased. The findings also did not reflect cases of follow-up loss. This may have resulted in a selection bias because the death group showed a higher incidence of comorbidities than the survival group. The small number of patients means that the findings are statistically less supported. Third, 11 patients who performed sacroplasty were not confirmed as osteoporosis with DEXA examinations. However, when we analyzed the cause that came to hospital, five patients were referred to minor trauma due to slip down and six patients visited without mention of special traumatic events. None of the patients had a major traumatic event with high force. These results were not significantly different from those of 29 patients who confirmed as osteoporosis on the DEXA examinations; slip down $(\mathrm{n}=17)$, no specific trauma $(\mathrm{n}=11)$, blunt trauma from the traffic accident $(\mathrm{n}=1)$. Given this, it is believed that osteoporotic sacral fractures were likely to have occurred in most of the patients in this study, including 11 patients who were not confirmed with DEXA examinations. This study was also limited in that the follow-up interval was not constant. The follow-up period varied from patient to patient, rather than to a specific point in time. Depending on the point of view, it may be difficult to interpret these data as true long-term outcomes. However, the findings are meaningful in that this was a follow-up study conducted for at least 1 year in all patients. Further studies addressing these shortcomings with a larger number of patients or prospective study designs are required.

\section{CONCLUSION}

PSP is an effective treatment for SIFs with positive longterm outcomes and an excellent success rate. Combined compression fractures in the thoracolumbar spine and degenerative lumbar pathologies could be possible poor outcome predictors. Since the remaining life expectancy of patients receiving sacroplasty is short, these possible factors that may affect the quality of the remaining life are important.

\section{CONFLICTS OF INTEREST}

No potential conflict of interest relevant to this article was reported.

\section{INFORMED CONSENT}

This type of study does not require informed consent.

\section{AUTHOR CONTRIBUTIONS}

Conceptualization : JL, EL, JWL

Data curation : JL, EL

Formal analysis : JL, EL

Methodology : EL, JWL, YK, JMA

Project administration : EL, JWL, YK, JMA

Visualization : JL

Writing - original draft : JL, EL

Writing - review \& editing: JL, EL, JWL, YK, JMA, HSK

\section{ORCID}

$\begin{array}{ll}\text { Jaehyung Lee } & \text { https://orcid.org/0000-0002-3998-6570 } \\ \text { Eugene Lee } & \text { https://orcid.org/0000-0003-4205-2362 } \\ \text { Joon Woo Lee } & \text { https://orcid.org/0000-0002-7106-5229 } \\ \text { Yusuhn Kang } & \text { https://orcid.org/0000-0003-1838-2564 } \\ \text { Joong Mo Ahn } & \text { https://orcid.org/0000-0002-1157-0020 } \\ \text { Heung Sik Kang } & \text { https://orcid.org/0000-0002-7024-388X }\end{array}$ 


\section{- Acknowledgements}

This work was supported by (grant No. 02-2018-039) from the SNUBH Research Fund.

\section{References}

1. Barre L, Lutz GE, Southern D, Cooper G : Fluoroscopically guided caudal epidural steroid injections for lumbar spinal stenosis: a restrospective evaluation of long term efficacy. Pain Physician 7 : 187-193, 2004

2. Bastian JD, Keel MJ, Heini PF, Seidel U, Benneker LM : Complications related to cement leakage in sacroplasty. Acta Orthop Belg 78 : 100105, 2012

3. Bellabarba C, Schroeder GD, Kepler CK, Kurd MF, Kleweno CP, Firoozabadi $R$, et al. : The AOSpine sacral fracture classification. Global Spine J 6 (1_suppl) : s-0036-1582696-s-0036-1582696, 2017

4. Eichler K, Zangos S, Mack MG, Marzi I, Vogl TJ : Outcome of longaxis percutaneous sacroplasty for the treatment of sacral insufficiency fractures with a radiofrequency-induced, high-viscosity bone cement.

Skeletal Radiol 43 : 493-498, 2014

5. Frey ME, Depalma MJ, Cifu DX, Bhagia SM, Carne W, Daitch JS : Percutaneous sacroplasty for osteoporotic sacral insufficiency fractures: a prospective, multicenter, observational pilot study. Spine J $8: 367-373$, 2008

6. Frey ME, Warner C, Thomas SM, Johar K, Singh H, Mohammad MS, et al. : Sacroplasty: a ten-year analysis of prospective patients treated with percutaneous sacroplasty: literature review and technical considerations. Pain Physician 20 : E1063-E1072, 2017

7. Gjertsen 0 , Schellhorn T, Nakstad PH : Fluoroscopy-guided sacroplasty: special focus on preoperative planning from three-dimensional computed tomography. Acta Radiol 49 : 1042-1048, 2008

8. Goode AP, Carey TS, Jordan JM : Low back pain and lumbar spine osteoarthritis: how are they related? Curr Rheumatol Rep $15:$ 305, 2013

9. Grogan J, Nowicki BH, Schmidt TA, Haughton VM : Lumbar facet joint tropism does not accelerate degeneration of the facet joints. AJNR Am J Neuroradiol 18 : 1325-1329, 1997

10. Gupta AC, Chandra RV, Yoo AJ, Leslie-Mazwi TM, Bell DL, Mehta BP, et al. : Safety and effectiveness of sacroplasty: a large single-center experi- ence. AJNR Am J Neuroradiol 35 : 2202-2206, 2014

11. Kamel EM, Binaghi S, Guntern D, Mouhsine E, Schnyder P, Theumann N : Outcome of long-axis percutaneous sacroplasty for the treatment of sacral insufficiency fractures. Eur Radiol 19 : 3002-3007, 2009

12. Kang SE, Lee JW, Kim JH, Park KW, Yeom JS, Kang HS : Percutaneous sacroplasty with the use of C-arm flat-panel detector CT: technical feasibility and clinical outcome. Skeletal Radiol 40 : 453-460, 2011

13. Korean Statistical Information Service: Population dynamics and dynamics trend. Available at : http://kosis.kr/statHtml/statHtml.do?orgld=101\&tblld=DT_1B8000F\&vw_cd=MT_ZTITLE\&list_ $\mathrm{id}=\mathrm{A2} \_6 \&$ seqNo $=\&$ lang_mode $=$ ko\&language $=$ kor\&obj_var_ id=\&itm_id=\&conn_path=MT_ZTITLE

14. Kortman K, Ortiz O, Miller T, Brook A, Tutton S, Mathis J, et al. : Multicenter study to assess the efficacy and safety of sacroplasty in patients with osteoporotic sacral insufficiency fractures or pathologic sacral lesions. J Neurointerv Surg 5 : 461-466, 2013

15. Kushchayev SV, Glushko T, Jarraya M, Schuleri KH, Preul MC, Brooks $M L$, et al. : $A B C s$ of the degenerative spine. Insights Imaging 9 : 253274,2018

16. Lee GY, Lee JW, Choi HS, Oh KJ, Kang HS : A new grading system of lumbar central canal stenosis on MRI: an easy and reliable method. Skeletal Radiol 40 : 1033-1039, 2011

17. Lee S, Lee JW, Yeom JS, Kim KJ, Kim HJ, Chung SK, et al. : A practical MRI grading system for lumbar foraminal stenosis. AJR Am J Roentgenol 194 : 1095-1098, 2010

18. Pathria M, Sartoris DJ, Resnick D : Osteoarthritis of the facet joints: accuracy of oblique radiographic assessment. Radiology $164:$ 227-230, 1987

19. Pereira $L P$, Clarençon $F$, Cormier $E$, Rose $M$, Jean $B$, Le Jean $L$, et al. : Safety and effectiveness of percutaneous sacroplasty: a single-centre experience in 58 consecutive patients with tumours or osteoporotic insufficient fractures treated under fluoroscopic guidance. Eur Radiol 23 : 2764-2772, 2013

20. Schroeder GD, Kurd MF, Kepler CK, Krieg JC, Wilson JR, Kleweno CP, et al. : The development of a universally accepted sacral fracture classification: a survey of AOSpine and AOTrauma members. Global Spine J 6 : 686-694, 2016

21. Weishaupt $D$, Zanetti $M$, Boos $N$, Hodler $J$ : MR imaging and CT in osteoarthritis of the lumbar facet joints. Skeletal Radiol $28: 215-219$, 1999 